\title{
NOTE ON CLOSURE FOR ORTHOGONAL POLYNOMIALS
}

\section{J. SHOHAT}

In this short note we give a simple theorem concerning closure for orthogonal polynomials (OP). The importance of closure in the theory of OP, particularly in the study of expansions of functions in series of $\mathrm{OP}$, can hardly be overestimated.

The notations employed below are those of my monograph, Théorie générale des polynômes orthogonaux de Tchebicheff (Mémorial des Sciences Mathématiques, vol. 66, 1934), referred to as M.

We write generally

to signify that

$$
f(x) \sim \sum_{n=0}^{\infty} f_{n} \phi_{n}(x)
$$

$$
\begin{gathered}
f_{n}=\int_{a}^{b} f(x) \phi_{n}(x) d \psi, \quad n=0,1,2, \cdots, \\
\phi_{n}(x) \equiv \phi_{n}(x ; a, b ; d \psi), \\
\int_{a}^{b} \phi_{m}(x) \phi_{n}(x) d \psi=\delta_{m n}, \quad m, n=0,1,2, \cdots .
\end{gathered}
$$

The hereafter assumed existence of $\int_{a}^{b} f^{2}(x) d \psi$ implies, as is known, the convergence of the series $\sum_{n=0}^{\infty} f_{n}^{2}$. By closure we mean the following equality, Parseval's formula:

$$
\int_{a}^{b} f^{2}(x) d \psi=\sum_{n=0}^{\infty} f_{n}^{2}
$$

THEOREM. If closure holds for the "symmetric" sequence

$$
\left\{\phi_{n}(x ;-h, h ; d \psi)\right\}, \quad \psi(\infty)-\psi(x) \equiv \psi(-x)
$$

[or

$$
\left.\left\{\phi_{n}(x ;-h, h ; p(x))\right\}, \quad p(x) \equiv p(-x)\right]
$$

then each of the following two sequences of $O P$ is closed

$$
\begin{array}{cc}
\left\{\phi_{n}\left(x ; 0, h^{2} ; d \psi_{1}\right)\right\}, & \left\{\phi_{n}\left(x ; 0, h^{2} ; d \psi_{2}\right)\right\} \\
d \psi_{1}(x)=2 d \psi\left(x^{1 / 2}\right), & d \psi_{2}(x)=x d \psi_{1}(x)
\end{array}
$$

[or

Received by the editors October 2, 1941. 


$$
\begin{array}{cc}
\left\{\phi_{n}\left(x ; 0, h^{2} ; p_{1}(x)\right)\right\}, & \left\{\phi_{n}\left(x ; 0, h^{2} ; p_{2}(x)\right)\right\} \\
p_{1}(x)=x^{-1 / 2} p\left(x^{1 / 2}\right), & \left.p_{2}(x)=x p_{1}(x)\right],
\end{array}
$$

and conversely.

It suffices to give the proof for $d \psi(x)$ proper, for the case where $d \psi(x)=p(x) d x$ is quite similar.

We have, for any $n=0,1,2, \cdots$ [M, pp. 19-20],

$$
\begin{aligned}
\phi_{2 n}(x ;-h, h ; d \psi) & \equiv \phi_{n}\left(x^{2} ; 0, h^{2} ; d \psi_{1}\right), \\
\phi_{2 n+1}(x ;-h, h ; d \psi) & \equiv x \phi_{n}\left(x_{n}{ }^{2} ; 0, h^{2} ; d \psi_{2}\right) .
\end{aligned}
$$

This yields at once, for any $n=0,1,2, \cdots$,

$$
\begin{aligned}
f_{2 n} & =\int_{-h}^{h} f(x) \phi_{2 n}(x ;-h, h ; d \psi) d \psi \\
& =\int_{0}^{h^{2}} F_{1}(x) \phi_{n}\left(x ; 0, h^{2} ; d \psi_{1}\right) d \psi_{1} \equiv f_{n, 1}, \\
f_{2 n+1} & =\int_{-h}^{h} f(x) \phi_{2 n+1}(x ;-h, h ; d \psi) d \psi \\
& =\int_{0}^{h^{2}} F_{2}(x) \phi_{n}\left(x ; 0, h^{2} ; d \psi_{2}\right) d \psi_{2} \equiv f_{n, 2},
\end{aligned}
$$

where

$$
F_{1}\left(x^{2}\right)=\frac{f(x)+f(-x)}{2}, \quad x F_{2}\left(x^{2}\right)=\frac{f(x)-f(-x)}{2} .
$$

We have further

$$
f(x)=F_{1}\left(x^{2}\right)+x F_{2}\left(x^{2}\right) \sim \sum_{n=0}^{\infty} f_{n} \phi_{n}(x ;-h, h ; d \psi) .
$$

Making use of (3), (4) and (5), we get readily

$$
\begin{aligned}
F_{1}\left(x^{2}\right) & \sim \sum_{n=0}^{\infty} f_{2 n} \phi_{2 n}(x ;-h, h ; d \psi), \\
x F_{2}\left(x^{2}\right) & \sim \sum_{n=0}^{\infty} f_{2 n+1} \phi_{2 n+1}(x ;-h, h ; d \psi), \\
F_{1}(x) & \sim \sum_{n=0}^{\infty} f_{n, 1} \phi_{n}\left(x ; 0, h^{2} ; d \psi_{1}\right), \\
F_{2}(x) & \sim \sum_{n=0}^{\infty} f_{n, 2} \phi_{n}\left(x ; 0, h^{2} ; d \psi_{2}\right),
\end{aligned}
$$




$$
\begin{aligned}
\int_{-h}^{h} x F_{1}\left(x^{2}\right) F_{2}\left(x^{2}\right) d \psi= & \\
\int_{-h}^{h} f^{2}(x) d \psi-\sum_{n=0}^{\infty} f_{n}^{2}= & {\left[\int_{0}^{h^{2}} F_{1}^{2}(x) d \psi_{1}-\sum_{n=0}^{\infty} f_{n, 1}^{2}\right] } \\
+ & {\left[\int_{0}^{h^{2}} F_{2}^{2}(x) d \psi_{2}-\sum_{n=0}^{\infty} f_{n, 2}^{2}\right] . }
\end{aligned}
$$

By Bessel's inequality, both brackets on the right side of (7) are nonnegative, and this directly proves the theorem stated.

REMARK. In the above discussion $h$ may be assumed to be infinite, for closure always holds for a finite interval.

The most interesting application of the foregoing is to Hermite and Laguerre polynomials. In fact, it follows from the above theorem that the closure of both sequences

$$
\left\{\phi_{n}\left(x ; 0, \infty ; e^{-x} x^{\alpha-1}\right)\right\}, \quad\left\{\phi_{n}\left(x ; 0, \infty ; e^{-x} x^{\alpha}\right)\right\}, \quad \alpha \geqq 1 / 2,
$$

implies that of $\left\{\phi_{n}\left(x ;-\infty, \infty ; e^{-x^{2}}|x|^{2 \alpha-1}\right)\right\}$, and conversely. In particular, taking $\alpha=1 / 2$, we conclude that the closure of Hermite polynomials $\left\{\phi_{n}\left(x ;-\infty, \infty ; e^{-x^{2}}\right)\right\}$ implies that either of the two sequences of Laguerre polynomials $\left\{\phi_{n}\left(x ; 0, \infty ; e^{-x} x^{-1 / 2}\right)\right\},\left\{\phi_{n}(x ; 0, \infty\right.$; $\left.\left.e^{-x} x^{1 / 2}\right)\right\}$ is closed, and conversely.

The University of Pennsylvania 